\title{
Quantitative Morphology Characterization of New PC-siloxane Copolymer Blends
}

\author{
Lanti Yang, Robert van de Grampel, Ramon Groote, Olivier Guise
}

SABIC Innovative Plastics, Department Technology and Innovation (T\&I), Bergen op Zoom, the Netherlands.

SABIC recently developed polycarbonate-polysiloxane (PC-siloxane) copolymer blends which offer unique combinations of chemical resistance and low temperature impact ductility compared to polycarbonate (PC) homo-polymers. These novel blends have additional excellent aesthetic properties at high polysiloxane levels in the blend, especially when compared to polycarbonate-polysiloxane copolymers with the same polysiloxane content [1,2]. In PC-siloxane copolymer blends, siloxane forms nano-domains and is dispersed in the PC matrix. As the siloxane nano-domain size and the dispersion have a significant influence on the material properties, it is critical to develop quantitative morphology characterization $[3,4]$ of PC-siloxane copolymer blends to offer a complete understanding of the relationship between material structure and properties.

This paper describes the new methods developed for the quantitative morphology characterization of PC-siloxane copolymer blends using a combination of atomic force microscopy (AFM) and image analysis to further understand the influence of morphology on the aesthetic properties. As shown in figure 1, conventional TEM approach can provide morphology images of PC-siloxane copolymer materials. However, it is very challenging to perform quantitative image analysis on the TEM images due to the lack of a strongly defined contrast between siloxane and the PC phase. As a result image analysis tends to underestimate the true size of the siloxane domains and offers a skewed size distribution. As the siloxane phase is softer than the PC phase, one can take advantage of AFM tapping mode imaging to yield an enhanced contrast between siloxane and the PC phase (figure 1) vs. TEM. This high contrast morphology imaging mode - based on local hardness of the material - enables the possibility of quantitative morphology studies on different PC-siloxane copolymer materials.

PC-siloxane blends were developed using a mixture of two types of PC-siloxane copolymers: opaque BPA-PDMS-1 resin (figure 1a) and transparent BPA-PDMS-2 resin (figure 1b). These two copolymers significantly differ in their chain architecture and, as a consequence in their morphology. As shown in figure 1, in transparent PC-siloxane copolymer (BPA-PDMS-2) siloxane is better dispersed in the PC matrix and exhibits smaller domains. To better understand the material structure and how it relates to its properties, siloxane nano-domains were quantitatively measured by combining AFM imaging and image analysis in a fully automated fashion. As shown in figure 2, the median size value of siloxane domains of "BPA-PDMS-2" and "BPA-PDMS-1"are compared by the Mann-Whitney test (because both size distribution are non-normal) and show statistically significant difference. Similar quantitative morphology measurements were performed on two PC-siloxane blends with different percentage of BPA-PDMS-1 and BPA-PDMS-2 resins. The two PC-siloxane copolymers blends have significantly smaller siloxane domains than BPA-PDMS-1 resin, approaching the domain sizes of BPA-PDMS-2 resin. These initial results confirm correlation between the aesthetic properties of PC-copolymer resins and siloxane domain structure within the polycarbonate matrix. Also the results suggest that the transparent BPA-PDMS-2 resin has significant influence on the siloxane domain size, which explains the excellent aesthetic properties of PC-siloxane copolymer blends. Furthermore, a method based on domain dilation [2] is adapted to this study to quantify and analyze the dispersion of siloxane domains in 
the PC matrix and to understand the influence of siloxane domains size on siloxane dispersion. The quantitative morphology study on both the siloxane domain size distribution and the siloxane domain dispersion provides insight on the relationship between the material structure and properties and significantly supports further improvements in materials properties.

\section{References:}

[1] Grampel, Robert van de et al., ANTEC 2014 conference proceeding, accepted.

[2] Venderbosch, Robert et al., Patent number: WO 2004/076541.

[3] O. Guise et al., Microscopy and Microanalysis, 17, S2, 2011, 1472.

[4] O. Guise et al., Microscopy and Microanalysis, 16, S2, 2010, 750.
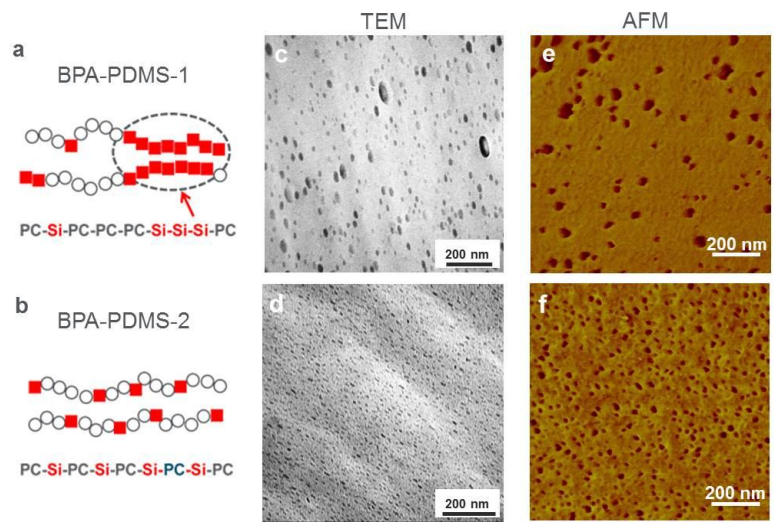

Figure 1. Chain architecture of opaque BPA-PDMS-1 resin (a) and transparent BPA-PDMS-2 resin (b). Morphology of "BPA-PDMS-1" by TEM (c) and AFM tapping mode imaging (e). Morphology of "BPA-PDMS-2" by TEM (d) and AFM tapping mode imaging (f). $Z$ scale range in AFM is $80^{\circ}$.
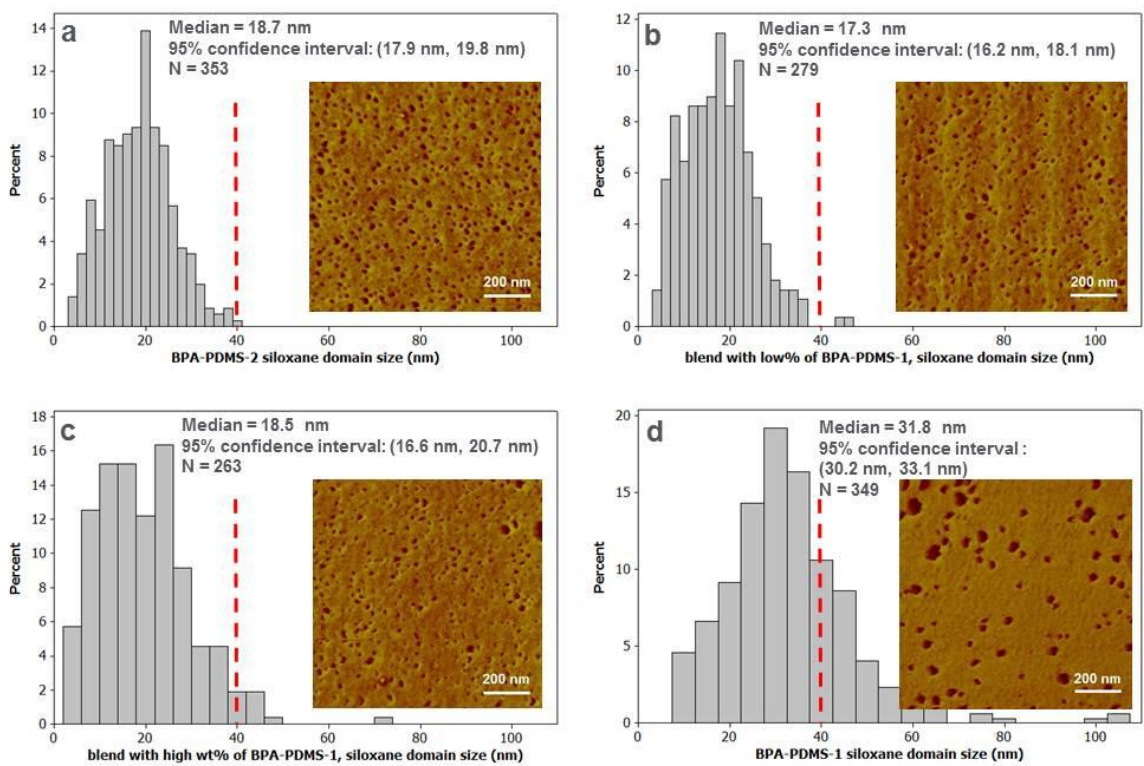

Figure 2. Quantitative morphology measurements of siloxane domain size based on AFM $1 \times 1 \mu \mathrm{m}$ scanning size: a) BPA-PDMS-2, (b) blend with low\% of BPA-PDMS-1, (c) blend with high wt $\%$ of BPA-PDMS-1 and (d) BPA-PDMS-1. Dashed red lines are made in all images at $40 \mathrm{~nm}$ to indicate the difference of domain size. Typical AFM $1 \times 1 \mu \mathrm{m}$ scanning size images are inserted. 